\title{
SEVERAL IDENTITIES IN STATISTICAL MECHANICS
}

\section{D. HIRSCHHORN}

Department of Pure Mathematics University of New South Wales

P.O. Box 1, Kensington

New South Wales 2033, Australia

(Received March 16, 1993)

\begin{abstract}
In an earlier paper concerning a solvable model in statistical mechanics, Miwa and Jimbo state a theta-function identity which they have checked to the 200th power, but of which they do not have a proof. The main objective of this note is to provide such a proof.
\end{abstract}

KEY WORDS AND PHRASES. $q$-series identity, Jacobi's triple product, sums and products. 1991 AMS SUBJECT CLASSIFICATION CODE(S). 83B23.

\section{INTRODUCTION.}

In their paper [1], Miwa and Jimbo state a theta function identity, which they have checked to the 2000th power, but of which they do not have a proof. They also state, and prove, two other theta function identities. The object of this note is to provide a proof of the first identity, and to make the observation that the other two identities are special cases of an identity of mine that generalizes the quintuple-product identity.

\section{MAIN RESULTS.}

Our only tool in proving the first identity is Jacobi's triple product identity,

$$
\left(-a q ; q^{2}\right)_{\infty}\left(-a^{-1} q ; q^{2}\right)_{\infty}\left(q^{2} ; q^{2}\right)_{\infty}=\sum a^{n} q^{n^{2}} .
$$

[Here, $(a: q)_{\infty}=\prod_{n \geq 1}\left(1-a q^{n-1}\right)$. and throughout this paper all sums are taken from $-\infty$ to $\infty$.]

The identity to be proved ([1], (B.13)) is

$$
\begin{aligned}
& \left(\sum q^{\left(3 n^{2}-3 n\right) / 2}\right)\left(\sum(-1)^{n} q^{6 n^{2}-4 n+1}\right)+\left(\sum q^{\left(3 n^{2}-n\right) / 2}\right)\left(\sum(-1)^{n} q^{6 n^{2}}\right) \\
= & \left(\sum q^{n^{2}}\right)\left(\sum(-1)^{n} q^{6 n^{2}-n}+\sum(-1)^{n} q^{6 n^{2}-5 n+1}\right)
\end{aligned}
$$

Our first step is to make use of (2.1) to express all the sums in (2.2) as products.

Thus

$$
\begin{aligned}
\sum q^{\left(3 n^{2}-3 n\right) / 2} & =2\left(-q^{3} ; q^{3}\right)_{\infty}^{2}\left(q^{3} ; q^{3}\right)_{\infty} \\
\sum(-1)^{n} q^{6 n^{2}-4 n} & =\left(q^{2}: q^{12}\right)_{\infty}\left(q^{10} ; q^{12}\right)_{\infty}\left(q^{12} ; q^{12}\right)_{\infty} \\
\sum q^{\left(3 n^{2}-n\right) / 2} & =\left(-q: q^{3}\right)_{\infty}\left(-q^{2}: q^{3}\right)_{\infty}\left(q^{3}: q^{3}\right)_{\infty} \\
\sum(-1)^{n} q^{6 n^{2}} & =\left(q^{6}: q^{12}\right)_{\infty}^{2}\left(q^{12}: q^{12}\right)_{\infty}
\end{aligned}
$$




$$
\sum q^{n^{2}}=\left(-q ; q^{2}\right)_{\infty}^{2}\left(q^{2} ; q^{2}\right)_{\infty}
$$

and

$$
\begin{aligned}
\sum(-1)^{n} q^{6 n^{2}-n} & +\sum(-1)^{n} q^{6 n^{2}-5 n+1} \\
& =\sum(-1)^{n}(-q)^{\left(3 n^{2}-n\right) / 2} \\
& =\prod_{n \geq 1}\left(1-(-q)^{n}\right) \\
& =\left(-q ; q^{2}\right)_{\infty}\left(q^{2} ; q^{2}\right)_{\infty} .
\end{aligned}
$$

So $(2.2)$ is equivalent to

$$
\begin{aligned}
& \left(-q ; q^{2}\right)_{\infty}^{3}\left(q^{2} ; q^{2}\right)_{\infty}^{2} \\
& =\left(-q ; q^{3}\right)_{\infty}\left(-q^{2} ; q^{3}\right)_{\infty}\left(q^{3} ; q^{3}\right)_{\infty}\left(q^{6} ; q^{12}\right)_{\infty}^{2}\left(q^{12} ; q^{12}\right)_{\infty} \\
& +2 q\left(-q^{3} ; q^{3}\right)_{\infty}^{2}\left(q^{3} ; q^{3}\right)_{\infty}\left(q^{2} ; q^{12}\right)_{\infty}\left(q^{10} ; q^{12}\right)_{\infty}\left(q^{12} ; q^{12}\right)_{\infty} \\
& =\left(-q ; q^{6}\right)_{\infty}\left(-q^{2} ; q^{6}\right)_{\infty}\left(q^{3} ; q^{6}\right)_{\infty}\left(-q^{4} ; q^{6}\right)_{\infty}\left(-q^{5} ; q^{6}\right)_{\infty}\left(q^{6} ; q^{6}\right)_{\infty} \\
& \cdot \cdot\left(q^{6} ; q^{12}\right)_{\infty}^{2}\left(q^{12} ; q^{12}\right)_{\infty} \\
& +2 q\left(-q^{3} ; q^{6}\right)_{\infty}^{2}\left(q^{3} ; q^{6}\right)_{\infty}\left(-q^{6} ; q^{6}\right)_{\infty}^{2}\left(q^{6} ; q^{6}\right)_{\infty} \\
& \cdot \cdot\left(q^{2} ; q^{12}\right)_{\infty}\left(q^{10} ; q^{12}\right)\left(q^{10} ; q^{12}\right)_{\infty}\left(q^{12} ; q^{12}\right)_{\infty} .
\end{aligned}
$$

If we put $-q$ for $q$, we see that (2.3) is equivalent to

$$
\begin{aligned}
& \begin{array}{l}
\left(q ; q^{2}\right)_{\infty}^{3}\left(q^{2} ; q^{2}\right)_{\infty}^{2} \\
=\left(q ; q^{6}\right)_{\infty}\left(-q^{2} ; q^{6}\right)_{\infty}\left(-q^{3} ; q^{6}\right)_{\infty}\left(-q^{4} ; q^{6}\right)_{\infty}\left(q^{5} ; q^{6}\right)_{\infty}\left(q^{6} ; q^{6}\right)_{\infty} \\
\cdot\left(q^{3} ; q^{6}\right)_{\infty}^{2}\left(-q^{3} ; q^{6}\right)_{\infty}^{2}\left(q^{6} ; q^{6}\right)_{\infty}\left(-q^{6} ; q^{6}\right)_{\infty} \\
-2 q\left(q^{3} ; q^{6}\right)_{\infty}^{2}\left(-q^{3} ; q^{6}\right)_{\infty}\left(-q^{6} ; q^{6}\right)_{\infty}^{2}\left(q^{6} ; q^{6}\right)_{\infty} \\
\cdot\left(q ; q^{6}\right)_{\infty}\left(-q ; q^{6}\right)_{\infty}\left(q^{5} ; q^{6}\right)_{\infty}\left(-q^{5} ; q^{6}\right)_{\infty}\left(q^{6} ; q^{6}\right)_{\infty}\left(-q^{6} ; q^{6}\right)_{\infty}
\end{array}
\end{aligned}
$$

Next divide by

$$
\left(q ; q^{2}\right)_{\infty}=\left(q ; q^{6}\right)_{\infty}\left(q^{3} ; q^{6}\right)_{\infty}\left(q^{5} ; q^{6}\right)_{\infty}
$$

and use the fact that

$$
\left(q ; q^{2}\right)_{\infty}\left(-q ; q^{2}\right)_{\infty}\left(-q^{2} ; q^{2}\right)_{\infty}=\left(q ; q^{2}\right)_{\infty}(-q ; q)_{\infty}=\left(q ; q^{2}\right)_{\infty} \frac{\left(q^{2} ; q^{2}\right)_{\infty}}{(q ; q)_{\infty}}=\frac{(q ; q)_{\infty}}{(q ; q)_{\infty}}=1
$$

with $q^{3}$ for $q$, and we see that (2.4) is equivalent to

$$
\begin{aligned}
(q ; q)_{\infty}^{2} & =\left(-q^{2} ; q^{6}\right)_{\infty}\left(-q^{4} ; q^{6}\right)_{\infty}\left(q^{6} ; q^{6}\right)_{\infty}\left(-q^{3} ; q^{6}\right)_{\infty}^{2}\left(q^{6} ; q^{6}\right)_{\infty} \\
& -2 q\left(-q ; q^{6}\right)_{\infty}\left(-q^{5} ; q^{6}\right)_{\infty}\left(q^{6} ; q^{6}\right)_{\infty}\left(-q^{6} ; q^{6}\right)_{\infty}^{2}\left(q^{6} ; q^{6}\right)_{\infty}
\end{aligned}
$$

We now prove $(2.5)$

$$
(q ; q)_{\infty}^{2}=\sum(-1)^{r+s} q^{\left(3 r^{2}-r+3 s^{2}-s\right) / 2} .
$$

Split this sum into two, according to whether $r+s$ is even or odd. If $r+s$ is even, let $r=m+n, s=m-n$; if $r+s$ is odd, let $r=m+n+1, s=m-n$.

We obtain

$$
\begin{aligned}
(q ; q)_{\infty}^{2} & =\sum q^{\left(3(m+n)^{2}-(m+n)+3(m-n)^{2}-(m-n)\right) / 2} \\
& -\sum q^{\left(3(m+n+1)^{2}-(m+n+1)+3(m-n)^{2}-(m-n)\right) / 2}
\end{aligned}
$$




$$
\begin{aligned}
& =\sum q^{3 m^{2}-m+3 n^{2}}-\sum q^{3 m^{2}+2 m+3 n^{2}-3 n+1} \\
& =\left(-q^{2} ; q^{6}\right)_{\infty}\left(-q^{4}: q^{6}\right)_{\infty}\left(q^{6} ; q^{6}\right)_{\infty}\left(-q^{3} ; q^{6}\right)_{\infty}^{2}\left(q^{6} ; q^{6}\right)_{\infty} \\
& -2 q\left(-q ; q^{6}\right)_{\infty}\left(-q^{5} ; q^{6}\right)_{\infty}\left(q^{6} ; q^{6}\right)_{\infty}\left(-q^{6} ; q^{6}\right)_{\infty}^{2}\left(q^{6} ; q^{6}\right)_{\infty}
\end{aligned}
$$

as required.

The other two identities stated and proved by Miwa and Jimbo are ([1], (B.12))

$$
\begin{aligned}
\left(\frac{1}{2} \sum q^{\left(3 n^{2}-3 n\right) / 2}\right)\left(\sum q^{12 n^{2}}\right) & +\left(\sum q^{\left(3 n^{2}-n\right) / 2}\right)\left(\sum q^{12 n^{2}-8 n+1}\right) \\
= & \left(\sum q^{2 n^{2}-n}\right)\left(\sum q^{4 n^{2}-2 n}\right)
\end{aligned}
$$

and

$$
\begin{aligned}
\left(\frac{1}{2} \sum q^{\left(3 n^{2}-3 n\right) / 2}\right)\left(\sum q^{12 n^{2}-12 n+3}\right) & +\left(\sum q^{\left(3 n^{2}-n\right) / 2}\right)\left(\sum q^{12 n^{2}-4 n}\right) \\
& =\left(\sum q^{2 n^{2}-n}\right)\left(\sum q^{4 n^{2}-2 n}\right) .
\end{aligned}
$$

These can be written, respectively, as

$$
\begin{aligned}
& \left(\sum q^{2 n^{2}-n}\right)\left(\sum q^{4 n^{2}-2 n}\right) \\
& =\left(\sum q^{6 n^{2}-3 n}\right)\left(\sum q^{12 n^{2}}\right)+\left(\sum q^{6 n^{2}-n}+\sum q^{6 n^{2}-5 n+1}\right)\left(\sum q^{12 n^{2}-8 n+1}\right)
\end{aligned}
$$

and

$$
\begin{aligned}
& \left(\sum q^{2 n^{2}-n}\right)\left(\sum q^{4 n^{2}-2 n}\right) \\
& =\left(\sum q^{6 n^{2}-3 n}\right)\left(\sum q^{12 n^{2}-12 n+3}\right)+\left(\sum q^{6 n^{2}-n}+\sum q^{6 n^{2}-5 n+1}\right)\left(\sum q^{12 n^{2}-4 n}\right) .
\end{aligned}
$$

In product form, these become

$$
\begin{aligned}
& \left(-q ; q^{4}\right)_{\infty}\left(-q^{3} ; q^{4}\right)_{\infty}\left(q^{4} ; q^{4}\right)_{\infty}\left(-q^{2} ; q^{8}\right)_{\infty}\left(-q^{6} ; q^{8}\right)_{\infty}\left(q^{8} ; q^{8}\right)_{\infty} \\
& =\left(-q^{3} ; q^{12}\right)_{\infty}\left(-q^{9} ; q^{12}\right)_{\infty}\left(q^{12} ; q^{12}\right)_{\infty}\left(-q^{12} ; q^{24}\right)_{\infty}^{2}\left(q^{24} ; q^{24}\right)_{\infty} \\
& +q\left(-q^{5} ; q^{12}\right)_{\infty}\left(-q^{7} ; q^{12}\right)_{\infty}\left(q^{12} ; q^{12}\right)_{\infty}\left(-q^{4} ; q^{24}\right)_{\infty}\left(-q^{20} ; q^{24}\right)_{\infty}\left(q^{24} ; q^{24}\right)_{\infty} \\
& +q^{2}\left(-q ; q^{12}\right)_{\infty}\left(-q^{11} ; q^{12}\right)_{\infty}\left(q^{12} ; q^{12}\right)_{\infty}\left(-q^{4} ; q^{24}\right)_{\infty}\left(-q^{20} ; q^{24}\right)_{\infty}\left(q^{24} ; q^{24}\right)_{\infty}
\end{aligned}
$$

and

$$
\begin{aligned}
& \left(-q ; q^{4}\right)_{\infty}\left(-q^{3} ; q^{4}\right)_{\infty}\left(q^{4} ; q^{4}\right)_{\infty}\left(-q^{2} ; q^{8}\right)_{\infty}\left(-q^{6} ; q^{8}\right)_{\infty}\left(q^{8} ; q^{8}\right)_{\infty} \\
& =\left(-q^{5} ; q^{12}\right)_{\infty}\left(-q^{7} ; q^{12}\right)_{\infty}\left(q^{12} ; q^{12}\right)_{\infty}\left(-q^{8} ; q^{24}\right)_{\infty}\left(-q^{16} ; q^{24}\right)_{\infty}\left(q^{24} ; q^{24}\right)_{\infty} \\
& +q\left(-q ; q^{12}\right)_{\infty}\left(-q^{11} ; q^{12}\right)_{\infty}\left(q^{12} ; q^{12}\right)_{\infty}\left(-q^{8} ; q^{24}\right)_{\infty}\left(-q^{16} ; q^{24}\right)_{\infty}\left(q^{24} ; q^{24}\right)_{\infty} \\
& +2 q^{3}\left(-q^{3} ; q^{12}\right)_{\infty}\left(-q^{9} ; q^{12}\right)_{\infty}\left(q^{12} ; q^{12}\right)_{\infty}\left(-q^{24} ; q^{24}\right)_{\infty}^{2}\left(q^{24} ; q^{24}\right)_{\infty}
\end{aligned}
$$

Now, consider the identity $([2],(2))$

$$
\begin{aligned}
& \left(-a q ; q^{2}\right)_{\infty}\left(-a^{-1} q ; q^{2}\right)_{\infty}\left(q^{2} ; q^{2}\right)_{\infty}\left(-b q^{2} ; q^{4}\right)_{\infty}\left(-b^{-1} q^{2} ; q^{4}\right)_{\infty}\left(q^{4} ; q^{4}\right)_{\infty} \\
& =\left(-a b^{-1} q^{3} ; q^{6}\right)_{\infty}\left(-a^{-1} b q^{3} ; q^{6}\right)_{\infty}\left(q^{6} ; q^{6}\right)_{\infty}\left(-a^{2} b q^{6} ; q^{12}\right)_{\infty}\left(-a^{-2} b^{-1} q^{6} ; q^{12}\right)_{\infty}\left(q^{12} ; q^{12}\right)_{\infty} \\
& +a q\left(-a b^{-1} q^{5} ; q^{6}\right)_{\infty}\left(-a^{-1} b q ; q^{6}\right)_{\infty}\left(q^{6} ; q^{6}\right)_{\infty}\left(-a^{2} b q^{10} ; q^{12}\right)_{\infty}\left(-a^{-2} b^{-1} q^{2} ; q^{12}\right)_{\infty}\left(q^{12} ; q^{12}\right)_{\infty} \\
& +a^{-1} q\left(-a b^{-1} q ; q^{6}\right)_{\infty}\left(-a^{-1} b q^{5} ; q^{6}\right)_{\infty}\left(q^{6} ; q^{6}\right)_{\infty}\left(-a^{2} b q^{2} ; q^{12}\right)_{\infty}\left(-a^{-2} b^{-1} q^{10} ; q^{12}\right)_{\infty}\left(q^{12} ; q^{12}\right)_{\infty}
\end{aligned}
$$


If in (2.12), we set $q^{2}$ for $q$, then $a=q^{-1}, b=q^{2}$. we obtain (2.10), while if we set $q^{2}$ for $q$ then $a=q^{-1}, b=q^{-2}$, we obtain (2.11).

\section{REFERENCES}

1. JIMBO, M. and MIWA, T., A solvable model and related Rogers-Ramanujan type identities, Physica D 15 (1985), 335-353.

2. HIRSCHHORN, M.D., A generalization of the quintuple product identity, J. Austral. Math. Soc. (Series A) 44 (1988), 42-45. 


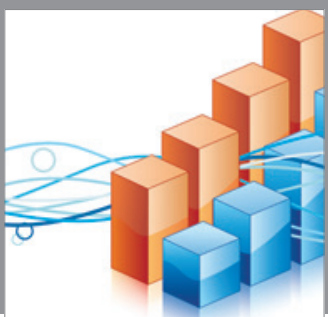

Advances in

Operations Research

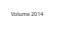

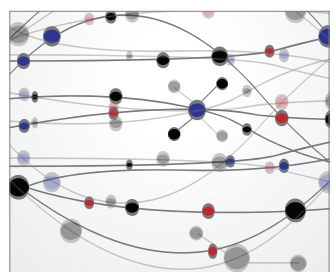

\section{The Scientific} World Journal
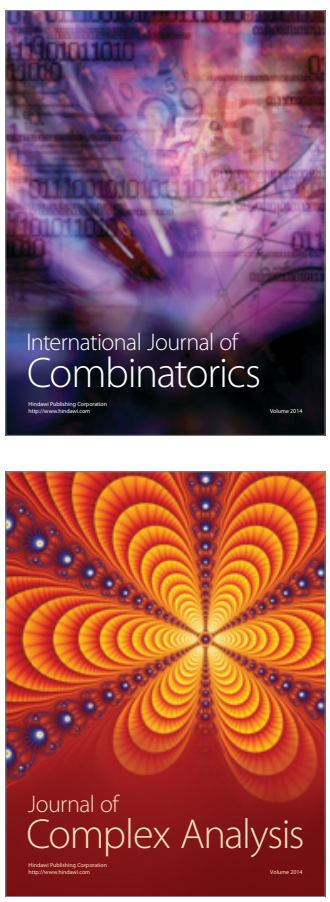

International Journal of

Mathematics and

Mathematical

Sciences
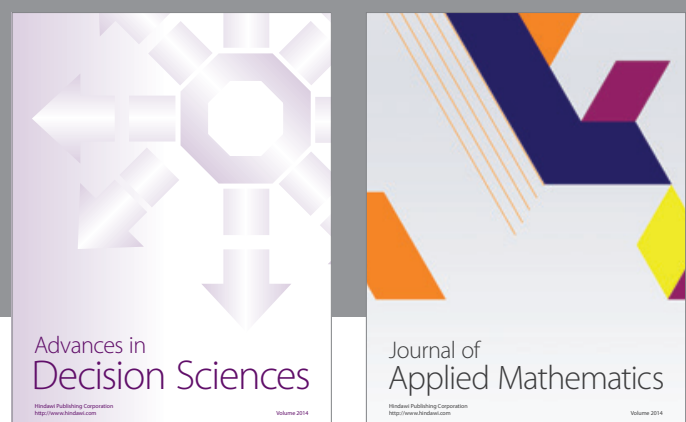

Journal of

Applied Mathematics
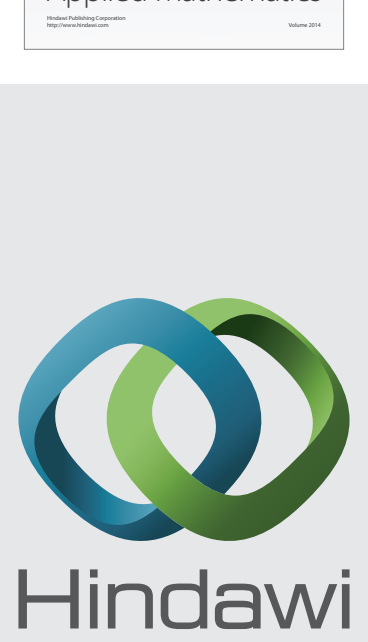

Submit your manuscripts at http://www.hindawi.com
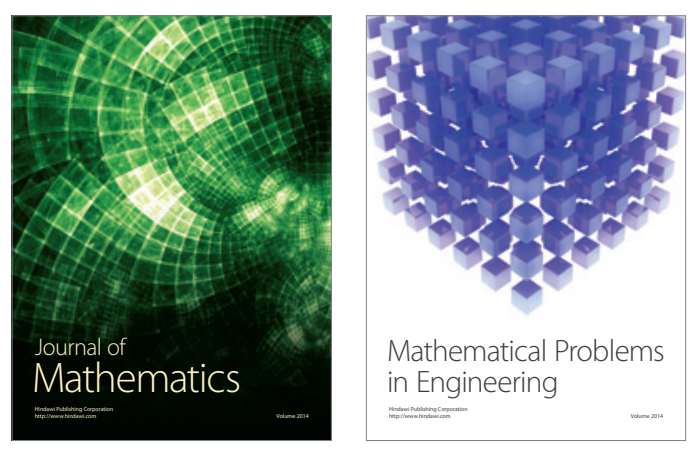

Mathematical Problems in Engineering
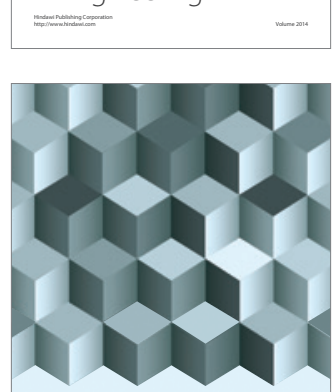

Journal of

Function Spaces
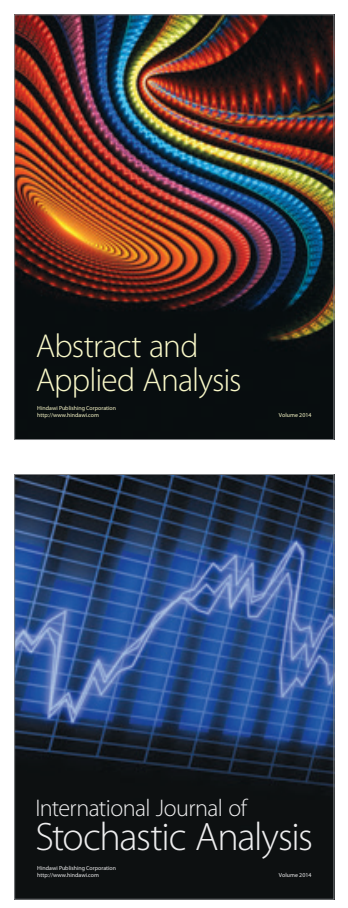

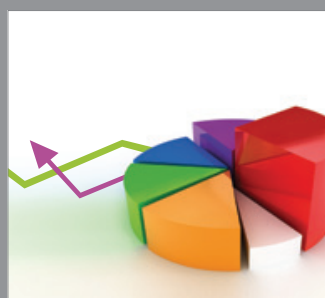

ournal of

Probability and Statistics

Promensencen
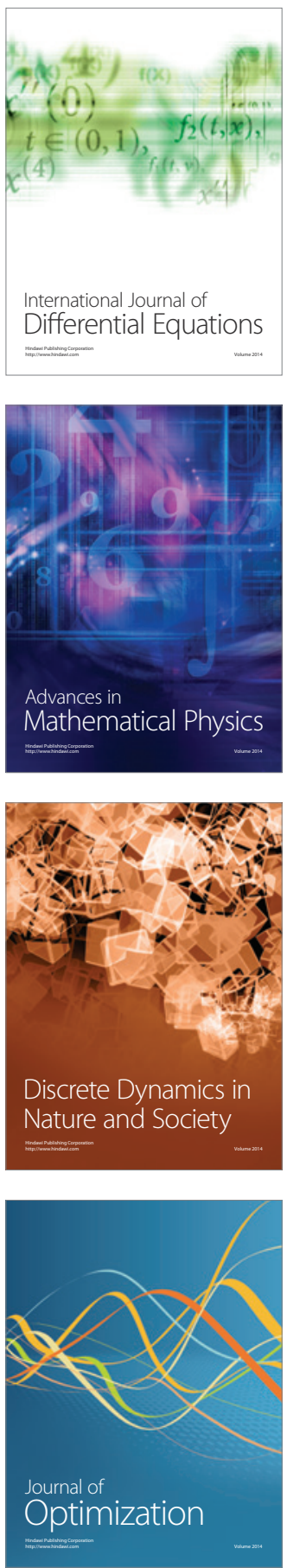